\title{
Microfluidic architectures for efficient generation of chemistry gradations in droplets
}

\author{
Judyta Wegrzyn • Adam Samborski • \\ Louisa Reissig • Piotr M. Korczyk • \\ Slawomir Blonski · Piotr Garstecki
}

Received: 15 May 2012/ Accepted: 2 August 2012/Published online: 28 August 2012

(C) The Author(s) 2012. This article is published with open access at Springerlink.com

\begin{abstract}
We demonstrate a strategy for construction of high-throughput microfluidic systems generating gradations of chemistry in micro-droplets. The productivity of the systems that we propose is limited only by the maximum rate of the droplet formation, and does not need to be limited by the rate of mixing. Multilayer polycarbonate chips transform two miscible input streams $A$ and $B$ into $\mathrm{N}$ streams of droplets, containing mixtures $[A]_{i},[B]_{i}$. Exemplary devices generate linear $\left([B]_{i} \propto i\right)$ and logarithmic gradations $\left(\ln [B]_{i} \propto i\right)$. We also analyze the use of the same strategy for the generation of concentration gradation in the streams of droplets comprising mixtures of liquids of different viscosities. The devices preserve the required distribution of compositions, while allowing the volume of the droplets to be tuned over almost two orders of magnitude (i.e. between 3 and $80 \mathrm{~nL}$ ).
\end{abstract}

Keywords Microfluidics - Generation of gradients . Droplet · Viscosity

Electronic supplementary material The online version of this article (doi:10.1007/s10404-012-1042-3) contains supplementary material, which is available to authorized users.

J. Wegrzyn · A. Samborski · P. M. Korczyk · P. Garstecki ( $\square)$ Institute of Physical Chemistry, Polish Academy of Sciences, Kasprzaka 44/52, 01-224 Warsaw, Poland

e-mail: garst@ichf.edu.pl

L. Reissig

Division of Biological Science, Graduate School of Science, Nagoya University, Nagoya 464-8602, Japan

P. M. Korczyk · S. Blonski

Institute of Fundamental Technological Research, Polish

Academy of Sciences, Pawińskiego 5B, 02-106 Warsaw, Poland

\section{Introduction}

Here we report a simple method-exploiting simple hydrodynamic laws - for the formation of parallel streams of droplets comprising predetermined composition gradations of two miscible input streams. The systems that we propose operate at the highest rate possible, i.e. at a rate that is limited only by the operation of the droplet generators, and not by the rate of mixing of the input streams. As we deliver the input streams directly to the droplet generators, our systems use the rapid mixing in the droplets to homogenize the mixtures. We characterize, in detail, the throughput of the devices and their performance measured in the fidelity of the generation of concentration gradations, and the tightness of the volume distribution of the droplets. Importantly, for preparatory applications that require throughput we also demonstrate the use of our system for the encapsulation of liquid mixtures of significantly dissimilar viscosities.

The low-to-moderate values of Reynolds numbers generate laminar flow and diffusional mixing (Kenis et al. 1999) making it possible to create controlled variations of chemical composition across the channels (Dertinger et al. 2001). The technique uses Kirchhoff's equations to design a network of channels that take two input streams $A$ and $B$, and then iteratively split, combine, and mix their flows to create $N$ outputs of predesigned combinations (Dertinger et al. 2002; Lin et al. 2004; Neils et al. 2004; Yamada et al. 2006; Irimia et al. 2006; Kim et al. 2008; Hattori et al. 2009; Lee et al. 2010; Campbell and Groisman 2007). All these techniques mix the sub-streams of $A$ and $B$ (and their combinations) within the network by diffusion, strongly limiting the maximum throughput. This strategy makes it also very difficult to create gradations from liquids of different viscosities as gradation of viscosity in the flow of 
combinations of the input streams requires additional linearization procedures (Yusuf et al. 2010).

The gradient-generating devices can be used to form streams of droplets exhibiting chemistry concentration gradations. For example, Niu et al. (2011) have applied a 'dilution chamber' to obtain, at high throughput, a set of droplets differing with concentration. Lorenz et al. (2008) have used the original (Dertinger et al. 2001) gradient generator to supply five dilutions of a fluorescent dye into five droplet generators. Damean et al. (2009) has used the outlets of a gradient-generating device (Campbell and Groisman 2007) as the source of the dispersed phase, supplying parallel droplet generators to form a linear gradation of fluorescein diphosphate, in order to screen the activity of the alkaline phosphatase. These reports add to the collection of techniques in droplet microfluidics oriented toward the screening of reaction conditions (Zheng et al. 2005; Churski et al. 2010; Cao et al. 2012; Miller et al. 2012) in analytical applications, where high throughput is not that important.

Preparatory applications of droplet microfluidics could greatly benefit from the simultaneous control of the volume of the droplets, and their composition. One example is the preparation of monodisperse polymeric particles or capsules, each having a well-defined profile of release of an active substance (Xu et al. 2009). In a broader view, the techniques for forming monodisperse droplets (Thorsen et al. 2001; Anna et al. 2003; Garstecki et al. 2005) have created a potential for a range of applications in the formulation of new materials, including particles (Xu et al. 2005) capsules (Utada et al. 2005) or even more complicated structures, such as multiple emulsions (Chu et al. 2007).

Although there exist both passive (Li et al. 2007; Nisisako and Torii 2008) and active (Guzowski et al. 2011) methods for high-throughput formation of monodisperse droplets in microfluidic systems of parallel droplet generators that can provide a throughput of e.g. $320 \mathrm{~mL} / \mathrm{h}$ (2.5 mL/h per junction) (Nisisako and Torii 2008; Song et al. 2006), there are no solutions that offer, simultaneously, high-throughput and control of the varied composition of the droplets in the parallel streams.

Here, we propose and detail a simple solution to the problem. It is well known that liquids can be rapidly mixed inside droplets (Song et al. 2006), and we, thus, propose a device in which the input streams have only one confluent point per droplet generator (immediately upstream of it), and that does not require mixing of the input solutions before they are enclosed inside a droplet. We first analyze the maximum throughput of the devices generating linear and logarithmic gradations of concentration in the droplets, and then examine the applicability of the same methodology for the generation of concentration gradations of liquids of varied viscosity in the droplets. In these demonstrations, we use chips fabricated in polycarbonate (PC), as this material is ideally suited to the fabrication of multilayer devices, presents compatibility with various organic oils that is superior to the commonly used polydimethylsiloxane (Lee et al. 2003), and can be modified to generate both water-in-oil (Jankowski et al. 2011) and oilin-water (Derzsi et al. 2011) emulsions.

\section{Materials and methods}

\subsection{Microfabrication}

We fabricated the chips via CNC micro milling (MSG4025, ErgWind, Poland). We then cleaned the milled slabs, and bonded the three layers together using registering pins and a method (Ogonczyk et al. 2010) that comprises swelling of the surface layer of PC with vapors of dichloromethane and subsequent compression $(0.2 \mathrm{MPa})$ of the plates at $130{ }^{\circ} \mathrm{C}$ for half an hour. The devices used for the formation of gradations of fractions of liquids of different viscosities were additionally modified with dodecylamine (Jankowski et al. 2011). While Fig. 1 gives a scheme of the channels on the chip, Figs. 2 and 8a show photographs of the three chips that we tested. Each of our microchips comprised three layers of polycarbonate (Macroclear, Bayer), two of which contained networks of microchannels [of uniform cross section $200 \times 200 \mu \mathrm{m}$ $( \pm 10 \mu \mathrm{m})]$. The middle layer comprised through-holes that communicated the outer layers.

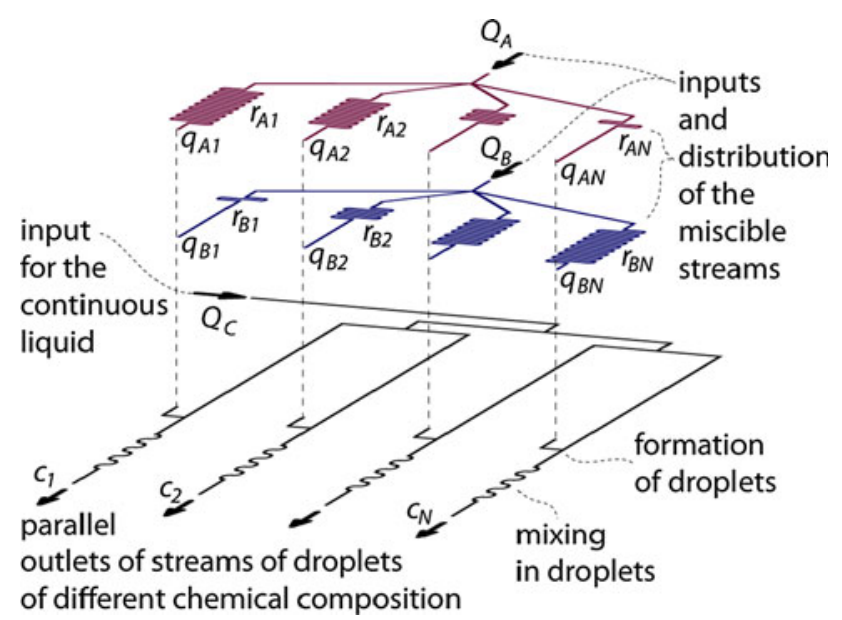

Fig. 1 Schematic diagram of the microfluidic system that distributes the inflow of two miscible streams $A$ and $B$, supplied at volumetric rates $Q_{A}$ and $Q_{B}$, into $N$ streams of liquid $A$ and $N$ streams of liquid $B$. The distribution of flow between streams $\left(\left\{q_{A_{i}}\right\}\right.$ and $\left.\left\{q_{B_{i}}\right\}\right)$ is determined by the set of hydraulic resistances $\left\{r_{A_{i}}\right\}$ and $\left\{r_{B_{i}}\right\}$. After the merging of each pair of streams, $N$ compound streams are transformed into droplets suspended in the continuous liquid, each stream having a predetermined concentration $[A]_{i}$ and $[B]_{i}$ 
Fig. 2 Photographs of the two microfluidic chips that we used to generate a linear (left) and logarithmic (right) variation of the concentration of a dye across the output channels $(c)$. The section responsible for the generation of the droplets and the region, where rapid mixing inside the droplets occurs, are marked as $a$ and $b$, respectively. For better visualization, we pumped red ink into the inlet for liquid $A$, blue ink into the inlet for liquid $B$ and green ink into the inlet for the continuous liquid
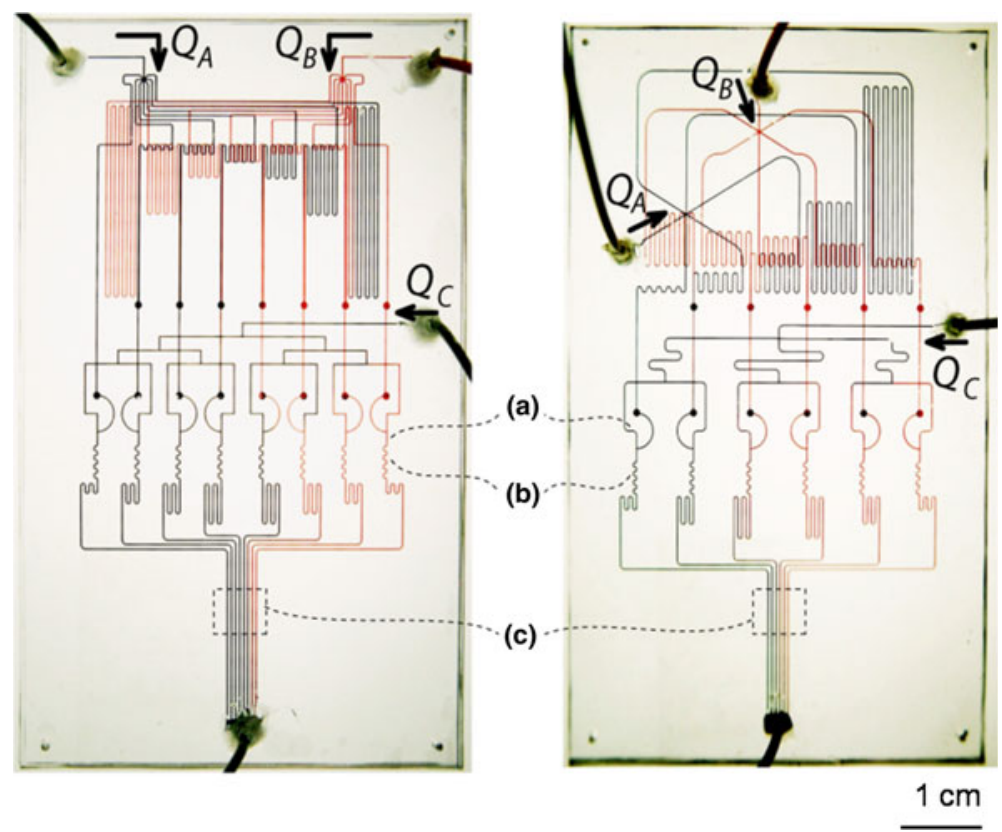

\subsection{Liquids}

We used distilled water (Millipore) or a $62 \%$ w/w solution of glycerol (Chempur, Poland) in distilled water (Millipore) and a $15 \%$ w/w mixture of ink (Quink, Parker Washable Blue or Encre Rouge, Waterman, France) in distilled water as the two input streams of the droplet phase. The viscosity of the applied solution of glycerin was 10 times higher than that of water. A network that distributes the continuous liquid was supplied by a $2 \%$ w/w solution of Span80 (Sigma-Aldrich, Germany) in hexadecane (Alpha Aesar, Germany).

\subsection{Experimental setup}

In the experiments engaging fluids of the same viscosity, we used syringe pumps (Harvard Apparatus, PHD2000) to infuse all liquids. In the case of the experiment based on testing the influence of differences in viscosities on the preservation of the gradient and droplet creation, we used a set of reducing valves and capillaries to control the values of the flow rates of the infused fluids $(Q)$ by varying the values of the applied pressure $(p)$. We established the dependence of $Q$ on $p$ during a calibration process. According to that, we could easily determine $Q$ on the basis of the applied $p$. We changed the length of the capillaries to preserve a constant ratio (1:1) of the infused fluids creating the droplet phase (Korczyk et al. 2011). In the experiments with liquids characterized by different viscosities, we used the pressure-driven flow in order to minimize the fluctuations of the rate of the inflow of the liquids into the chip. To acquire videos that we subsequently analyzed with a MatLab routine, we used a stereoscope (Nikon SMZ1500) equipped with a fast camera (Photron 1024).

\subsection{Theoretical model}

The chip is supplied with two miscible droplet phases, $A$ and $B$, and a continuous liquid, $C$, that preferentially wets the walls of the channels, and is not miscible either with $A$ or with $B$. We adopt the simplest possible topology for the networks of channels that distribute liquids $A$ and $B$ to the droplet generators (Fig. 1). Each inlet (of liquid $A$ and of liquid $B$ ) splits into $N$ channels $\left\{A_{i}\right\}$ and $\left\{B_{i}\right\}$. These channels are characterized by hydraulic resistances $\left\{r_{A_{i}}\right\}$ and $\left\{r_{B_{i}}\right\}$. Channels $A_{i}$ and $B_{i}$ join at a confluent point at the entrance to the inlet channel for the droplet phase in the $i$ th droplet generator, and supply it with liquids $A$ and $B$ at rates $q_{A_{i}}$ and $q_{B_{i}}$, respectively. The dimensionless concentration $\hat{C}_{i}$ of species $B$, defined as $C_{i} / C_{B}$, where $C_{B}$ is the concentration of species $B$ in the input stream $B$ in the $i$ th inlet is $\hat{C}_{i}=q_{B_{i}} / q$, where $q=q_{A_{i}}+q_{B_{i}}$. We set, as a requirement, that $q=\left(Q_{A}+Q_{B}\right) / N$, i.e. that the total rate of flow of the two droplet liquids through each droplet generator is the same. Importantly, the same strategy and calculations can be used to set the flow rates through each $\mathrm{T}$-junction to different values.

\subsection{Calculation of the lengths of the channels}

In order to design the microfluidic network of channels, we first set the desired combination of concentrations $\left\{\hat{C}_{i}\right\}$, and then calculate (1) the sets of resistances $\left(\left\{r_{A_{i}}\right\}\right.$ and $\left\{r_{B_{i}}\right\}$ ), and (2) the required ratio of the inflow rates of the two liquids $Q_{A} / Q_{B}$ that will yield the desired profile of concentrations. We first note that $Q_{X}=\sum_{i=1}^{N} q_{X_{i}}$, where $X=A$ or $B$. Each $q_{X_{i}}=\Delta p / r_{X_{i}}$, where $\Delta p$ is the difference 
of pressures at the inlet and at the terminus of the distribution channel (the $i$ th confluent point). From the definition of $\hat{C}_{i}$ we know that $q_{A_{i}}=q\left(1-\hat{C}_{i}\right)$ and $q_{B_{i}}=q \hat{C}_{i}$. Thus, $Q_{A} / Q_{B}=\left(\sum_{i=1}^{N} \hat{C}_{i}\right) /\left(N-\sum_{i=1}^{N} \hat{C}_{i}\right)$ yields the required ratio of inflow rates of the two miscible liquids. It can be shown (SI, Fig. 12) that $\Delta p$ is the same for all the distribution channels, provided that the resistances of the channels, running downstream from each confluent point via the droplet generator to the common outlet, are the same. This, via the Hagen Poiseuille law $Q=\Delta p / r$, allows us to write: $r_{A_{i}} / r_{A_{k}}=\left(1-\hat{C}_{k}\right) /\left(1-\hat{C}_{i}\right)$ and $r_{B_{i}} / r_{B_{k}}=\hat{C}_{k} / \hat{C}_{i}$, where indices $i$ and $k$ refer to any two of the parallel lines in the system. Since, for channels of constant (and the same) cross section, the hydraulic resistance is proportional to the length of the channel, we rewrite these in terms of lengths: $l_{A_{i}} / l_{A_{k}}=\left(1-\hat{C}_{k}\right) /\left(1-\hat{C}_{i}\right)$ and $l_{B_{i}} / l_{B_{k}}=C_{k} / \hat{C}_{i}$. Then it is enough to set an arbitrary length of one of the channels in each set $\left\{l_{A_{i}}\right\}$ and $\left\{l_{B_{i}}\right\}$ to calculate all the others.

We designed and fabricated two devices (Fig. 2) for the examination of their throughput: a system generating a linear gradation of $\hat{C}_{i}$, and a device producing a logarithmic variation of $\hat{C}_{i}$. In the 'linear' generator we set eight out channels for the generation of $\left\{\hat{C}_{i}\right\}=\{1,6 / 7,5 / 7,4 / 7,3 / 7$, $2 / 7,1 / 7,0\}$. Assuming an arbitrary reference length $l_{A_{1}}=26.28 \mathrm{~mm}$ we obtain $\left\{l_{A_{i}}\right\}=\{26.28,30.68,36.76$, 45.96, 61.32, 91.96, 183.96, infinity $\}(\mathrm{mm})$ and (setting $\left.l_{B_{8}}=26.26 \mathrm{~mm}\right)\left\{l_{B_{i}}\right\}=\{$ infinity, 183.96, 91.96, 61.32, $45.96,36.76,30.68,26.26\}(\mathrm{mm})$. In the 'logarithmic' chip we designed six outlet channels producing $\left\{\hat{C}_{i}\right\}=\{1,1 / 2$, $1 / 4,1 / 8,1 / 16,0\}$ with corresponding lengths of the distribution channels $\left\{l_{A_{i}}\right\}=\{50,53.33,57.14,66.67$, 100.00 , infinity $\}(\mathrm{mm})$ and $\left\{l_{B_{i}}\right\}=\{$ infinity, $320,160,80$, $40,20\}(\mathrm{mm})$. Obviously, the channels of 'infinite' length are designed to carry zero flow, and thus we simply do not incorporate them into the design. The layouts of the channel networks on each layer are given in Fig. 13 in the Supplementary Information.

\subsection{Operation of the system}

Apart from the distribution network for the liquids $A$ and $B$, the chip integrates several essential elements (Fig. 2): an inlet and distribution network for the continuous liquid, a set of channels between the confluent points and the T-junctions where liquids $A$ and $B$ flow together, a set of T-junction droplet generators, a set of meanders for mixing the content of the droplets, and a set of outlet channels that allow for simultaneous visualization of the streams of droplets. We supply the continuous liquid through a symmetric 'fractal' cascade of channels presenting equal hydraulic resistance for all $N$ lines.

\section{Results}

\subsection{Characterization of throughput}

\subsubsection{Single T-junction}

As a reference for the systems generating concentration gradations in droplets, we first examined the maximum throughput and the range of droplet volumes that can be generated in a single T-junction of the same dimensions as the T-junctions in the parallel systems. All the channels that constitute the T-junction are square $(200 \times 200 \mu \mathrm{m})$ in cross section and the side channel joins the main channel at a right angle. We used water as the droplet phase, and a $2 \%(w / w)$ solution of Span80 in hexadecane as the continuous liquid. The single $\mathrm{T}$-junction displays the scaling relation relevant to low values of capillary numbers (Garstecki et al. 2006; Christopher and Anna 2007; van Steijn et al. 2010) in which the volume ( $V$ ) of the droplets depends predominantly on the ratio of the flow rates of the droplet $\left(Q_{\mathrm{d}}\right)$, and of the continuous $\left(Q_{\mathrm{c}}\right)$ liquids (Fig. 3a). Figure $3 \mathrm{~b}$ shows the range of flow rates, within which the system produces droplets. Outside this range the device shifts either to a laminar co-flow (Guillot and Colin 2005; Guillot et al. 2009), or jetting (Lee et al. 2011) regimes. The maximum throughput of the droplet phase in a single junction is ca. $6 \mathrm{~mL} / \mathrm{h}$ corresponding to the generation of 174 drops/s. In the integrated systems, the maximum frequencies that we recorded were about 110 and 197 drops/s per single junction in the linear and logarithmic chips, respectively. The total throughput of the systems is higher as it encompasses the contributions from each parallel section: $882 \mathrm{drops} / \mathrm{s}$ in the linear and 1,179 drops/s in the logarithmic device. The system that used liquids of different viscosities presented lower productivities-317 and 40 drops/s in the whole device and in the single junction, respectively.
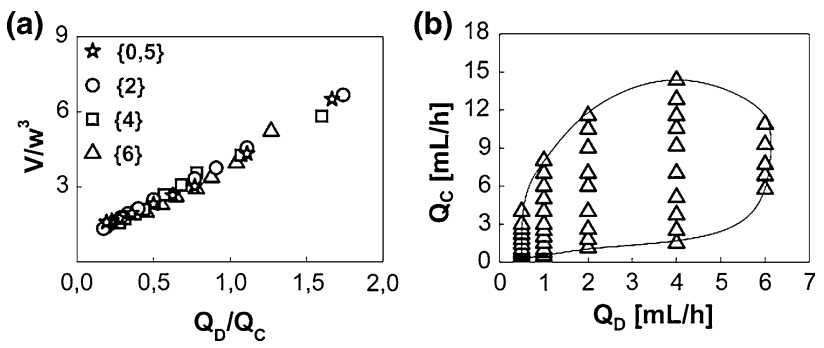

Fig. 3 Single T-junction. a Volume of droplets as a function of the ratio of flow rates [the numbers in curly brackets give the $Q_{D}$ in $(\mathrm{mL} / \mathrm{h})]$. b The range of operation of the T-junction in the dripping regime in the space spanned by the rates of flow 


\subsubsection{Volume of droplets produced in parallel}

We varied the total rate of flow of the droplet phase $\left(Q_{D}=Q_{A}+Q_{B}\right)$ and the rate of flow of the continuous $\left(Q_{C}\right)$ liquid to measure the range of operation and volume of the droplets formed in the parallel systems. In order to extensively probe the $\left(Q_{D}, Q_{C}\right)$ space, we first varied $Q_{C}$ for three constant values of $Q_{D}=\{6,16,30\}(\mathrm{mL} / \mathrm{h})$, and then varied $Q_{D}$ with $Q_{C}=\{2,5,10\}(\mathrm{mL} / \mathrm{h})$. This allowed us to determine the ranges of flow rates over which the systems produced droplets (Fig. 4).

Both microchips displayed similar ranges of operation to the single T-junction. We attribute the slight reduction of these ranges to small geometric differences between the junctions, resulting from imperfections in their fabrication.
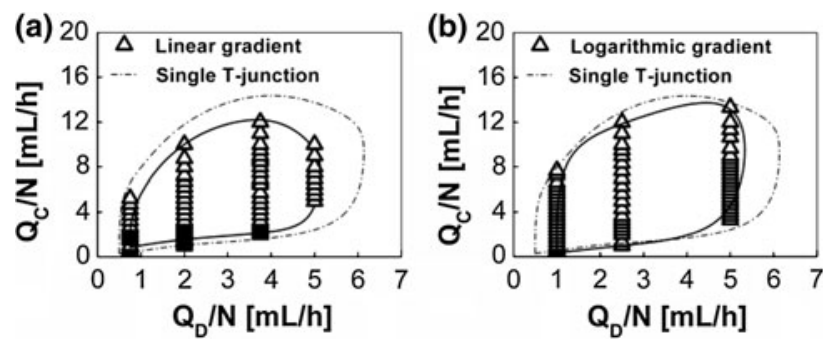

Fig. 4 The solid lines give the ranges of operation of the linear (a) and logarithmic (b) systems within which the devices produce droplets. For comparison the dashed line shows the working range of a single T-junction. The triangles show the experimental points from the parallel devices, and are values obtained per single T-junction
Also, the volume of the droplets varies with $Q_{D}$ and $Q_{C}$, similarly as in the case of the single T-junction. Figure 5 demonstrates these trends, together with exemplary micrographs of the droplets.

\subsubsection{Volume distribution of the droplets}

We characterized the tightness of the volume distribution of the droplets in the individual channels, and in the whole device (across channels). We calculated the standard deviation $\sigma$ of the lengths $L_{i}$ of the droplets within a channel $\sigma=\left[(1 / n) \sum_{i-1}^{n}\left(L_{i}-\bar{L}_{i}\right)^{2}\right]^{1 / 2}$ around the mean $\bar{L}=\left[(1 / n) \sum_{i-1}^{n} L_{i}\right]$. We found $\sigma$ to be always lower than $5 \%$ of $\bar{L}$, signifying tightly monodisperse populations within each channel.

To determine the tightness of the volume distribution across the parallel channels, we used the same formula and calculated the standard deviation $\left(\sigma^{*}\right)$ around the mean $\bar{L}^{*}$. We observed that, while at higher flow rates the emulsions are tightly monodisperse $\left(\sigma^{*} / \bar{L}^{*}<5 \%\right)$, at lower flow rates the variation of the droplet volumes in the parallel channels increases to $\sim 10 \%$, and, in some cases, exceeds this value (Fig. 6). We associate the higher standard deviation of the volumes between the channels in comparison to the volumes within each channel mostly to imperfections in the fabrication of the parallel systems.
Fig. 5 Dependence of the volume of the microdroplets on the ratio of flow rates of the droplet and continuous phases in the case of linear (a) and logarithmic (b) gradients. The numbers in curly brackets give $Q_{D}$ in $\mathrm{mL} / \mathrm{h}$. Micrographs illustrating the microdroplets of different size, depending on the applied rates of flow (left micrographs $Q_{D}=6 \mathrm{~mL} / \mathrm{h}$, $Q_{C}=55 \mathrm{~mL} / \mathrm{h} ;$ right micrographs $Q_{D}=6 \mathrm{~mL} / \mathrm{h}$, $\left.Q_{C}=2 \mathrm{~mL} / \mathrm{h}\right)$
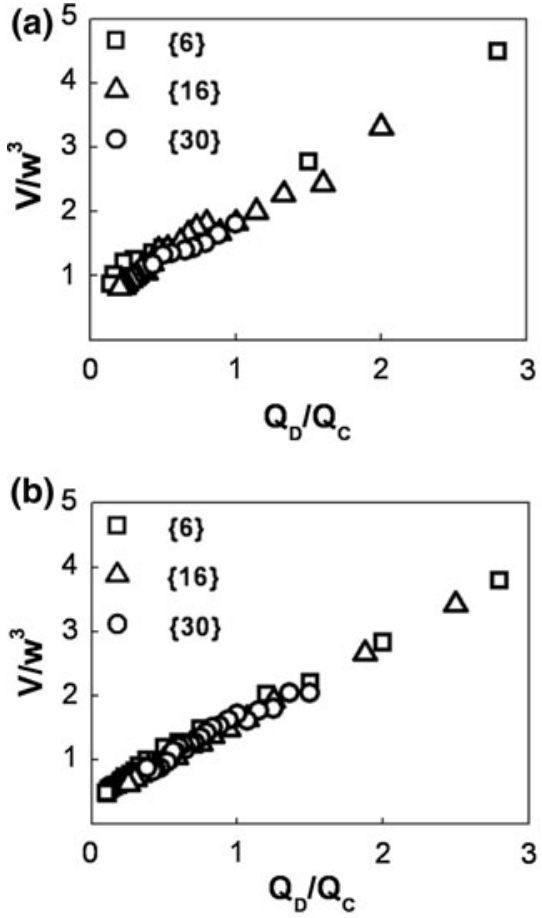
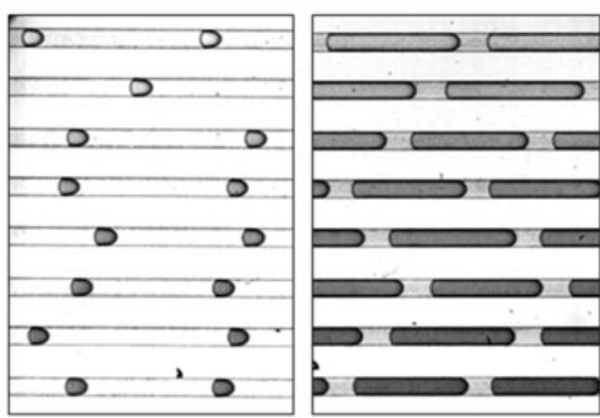

$500 \mu \mathrm{m}$

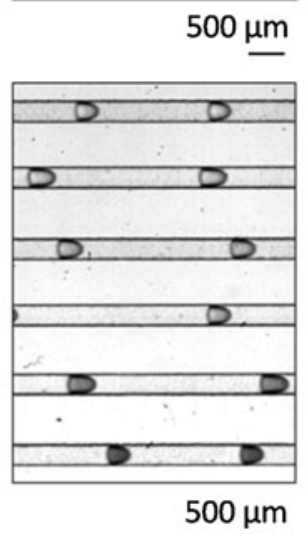

$500 \mu \mathrm{m}$

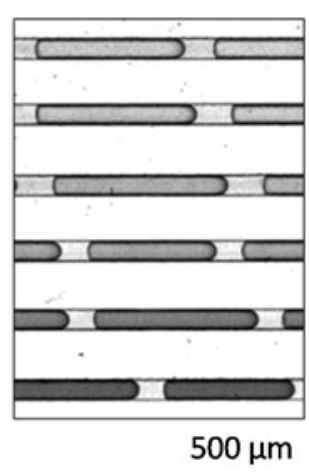



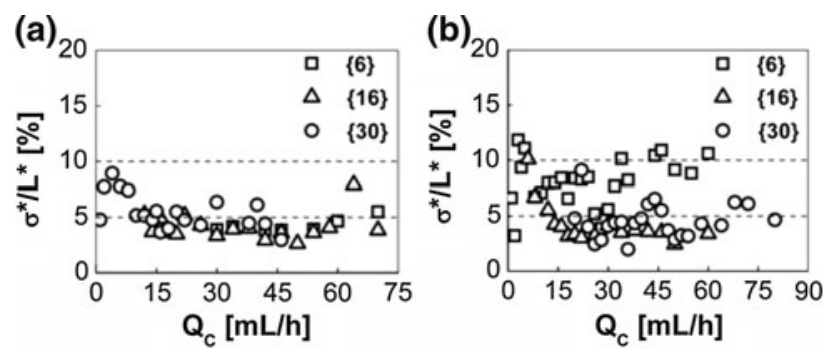

Fig. 6 The relative standard deviation of the droplet lengths across the microchannels for the linear (a) and logarithmic (b) devices. The different symbols correspond to different flow rates $\left\{Q_{D}\right\}(\mathrm{mL} / \mathrm{h})$

\subsection{Characterization of the concentration profile}

We used image analysis to extract the concentration of the dye in the droplets in the parallel outlet streams via the Lambert-Beer law $I=I_{0} e^{-a c l}$ where $I_{0}$ and $I$ are the intensities of the incident and recorded light, respectively, $\alpha$ the molar absorptivity of the dye, $l$ the length of the optical path through the droplet, and $C$ is the concentration of the dye. As $I_{0}, l$ and $\alpha$ are constant, we could assume that $C \propto-\ln I$. We normalized the such-determined concentrations to the 0-1 non-dimensional scale, where 1 describes the maximum concentration of the dye, and 0 neat water. Figure $7 \mathrm{a}, \mathrm{b}$ shows a perfect match of the recorded concentration gradation in the case of the linear devices, and a reasonable match for the logarithmic one.

In order to assess the fidelity of the generation of the prescribed concentration gradations quantitatively, we calculated the coefficient of determination $\left(R^{2}\right)$ for a wide range of flow rates. We used $R^{2}=1-(\mathrm{SSE} / \mathrm{SST})$, where the error sum of squares (SSE) is defined as $\mathrm{SSE}=\sum_{i=1}^{N}\left(C_{i}-\hat{C}_{i}\right)^{2}$
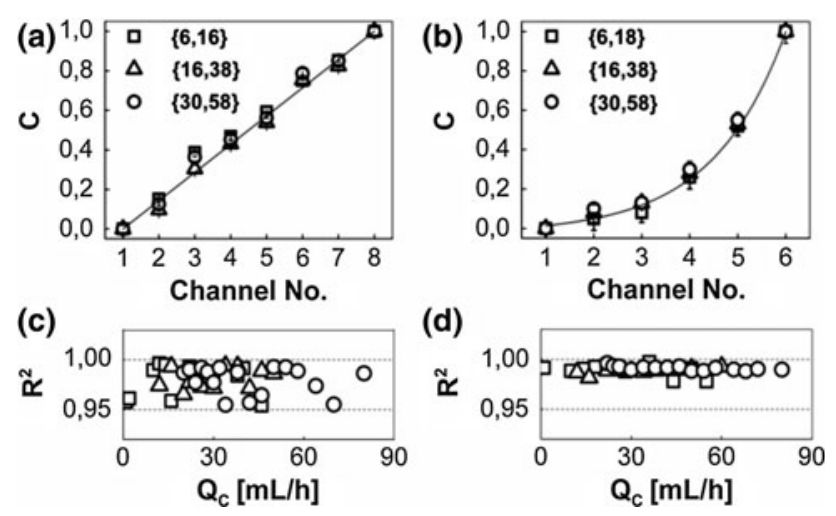

Fig. 7 The plots illustrate the correlation of the designed gradations of concentration (solid line) with the experimentally measured profiles in the linear (a) and logarithmic (b) devices. The symbols code for different flow rates $\left\{Q_{D}, Q_{C}\right\}$, given in $\mathrm{mL} / \mathrm{h}$. The dependence of $R^{2}$ on the flow rate of the continuous phase in the case of the linear (c), and logarithmic (d) gradients. The symbols on the plot code for different flow rates of the droplet phase $Q_{D}$ [squares 6 , triangles 16 and circles $30(\mathrm{~mL} / \mathrm{h})]$ and the total sum of squares (SST) $\mathrm{SST}=\left(\sum_{i=1}^{N} C_{i}^{2}\right)-$ $(1 / N)\left(\sum_{i=1}^{N} C_{i}\right)^{2}$ where $N$ is the number of channels, $C_{i}$ and $\hat{C}_{i}$ are the experimental and required values of the concentration, respectively. In all cases, these values were close to unity for practically all the measurements (Fig. 7c, d). This indicates that the systems generate the desired concentration gradations in the droplets over almost the whole range of flow rates that support the formation of droplets at all.

\subsection{Formation of concentration gradations} from the input streams of different viscosities

It is well known that the viscosity of the droplet phase affects both the operational range of the flow rates of droplet generators, and the tightness of the volume distribution of the droplets (Tice et al. 2004; Seo et al. 2005; Husny and Cooper-White 2006; Nie et al. 2008; Christopher et al. 2008; Berthier et al. 2010; Yeom and Lee 2011). For example, Berthier et al. (2010) have shown that liquids of higher viscosities produce smaller droplets. Also Tice et al. (2004) have shown that, in a T-junction, the increasing viscosity of the input droplet phase limits the range of rates of flow within which the system produces monodisperse droplets. The higher the viscosity of the inner phase, the sooner (i.e. at progressively smaller flow rates) the system shifts to the co-flow regime. The problem of droplet formation from a compound stream comprising lamellae of two liquids of two different viscosities has, so far, not been addressed.

In addition, the formation of concentration gradations of liquids of different viscosities is difficult in the standard microfluidic gradient generators (Yusuf et al. 2010). Mixing of combinations of liquids of different viscosities produces a gradation of viscosity that influences the distribution of the flow through a cascade of splitting and merging junctions in the networks. In such a system, in order to produce the required concentration gradation at the outlet, it is necessary to apply additional linearization procedures (Yusuf et al. 2010) that are specific to the particular set of liquids, and cannot be used for a different set.

Here we demonstrate that the architecture that we discussed above is suitable also for the formation of droplets presenting liquid content gradations of different viscosities. The strategy of the mixing of the input streams already after they are encapsulated into droplets, takes advantage of a more efficient mixing when the liquids that constitute a droplet differ in their viscosities (Tice et al. 2004).

We use the same general strategy in the design of the system-with the pairs of sub-streams $A$ and $B$ joining at a confluent point immediately upstream of the junction. In the chip for the formation of droplets comprising liquids of 
Fig. 8 a Photograph of the microfluidic chip that we used to generate a linear concentration gradation from fluids differing in their viscosity. We pumped red ink into the inlet for liquid $A(65 \%$ solution of glycerol in distilled water), blue ink into the inlet for liquid $B$ (distilled water), and green ink into the inlet for the continuous liquid (hexadecane with SPAN80). Insets $\mathbf{b}$ and c show two configurations for the delivery of the more viscous $(V)$ and less viscous $(N V)$ liquids to the $\mathrm{K}$-junction. Inset d shows a collection of micrographs of the K-junctions and the droplets immediately after their formation in the gradient-generating chip operated in the NV-V configuration (a)

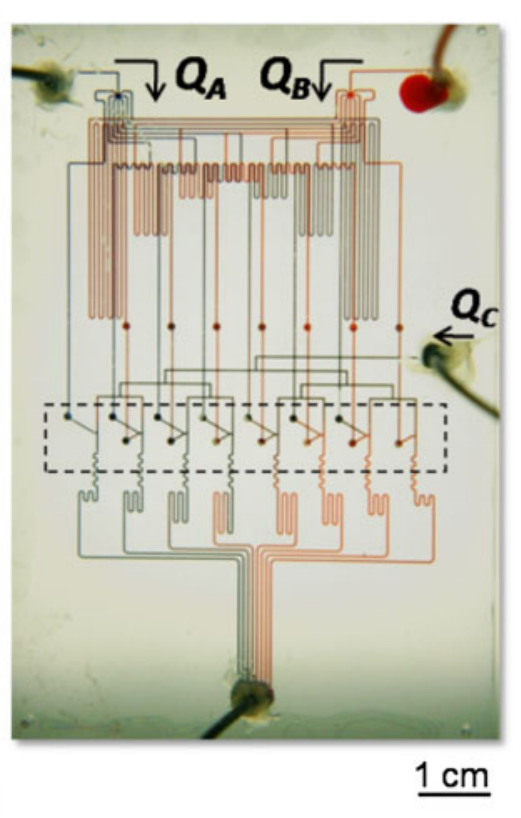

(b)

NV-V

(c)

V-NV

$\begin{array}{lll}a_{A} & Q_{B} & a_{C}\end{array}$

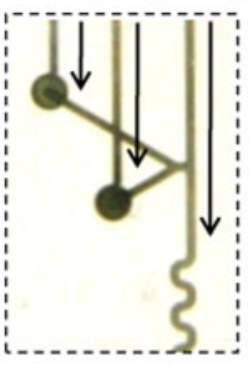

$a_{B} \quad a_{A} a_{C}$

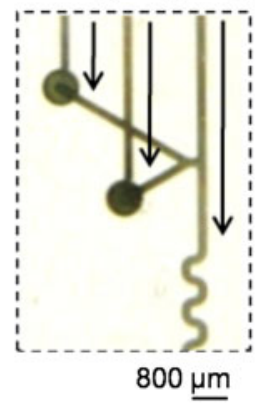

NV
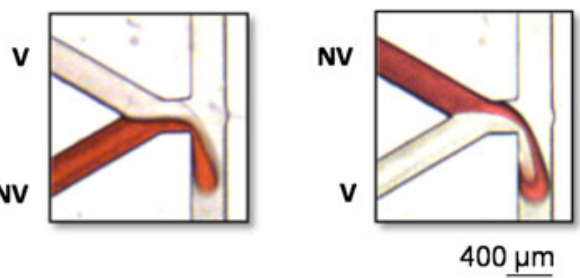

(d)

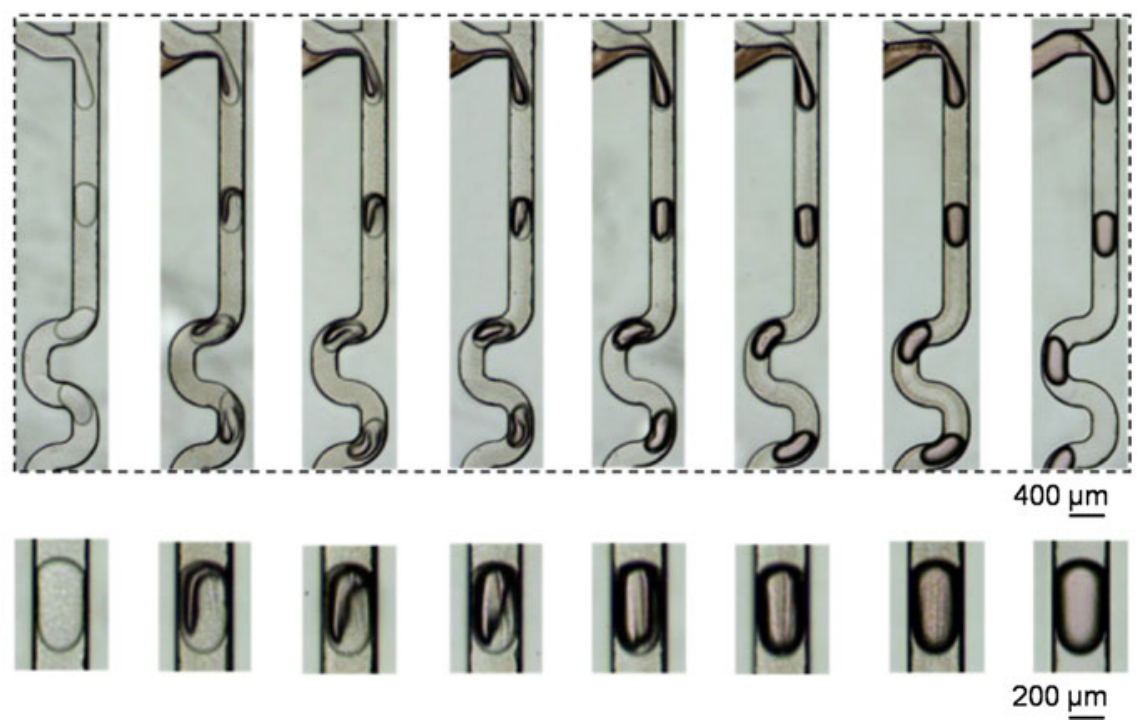

different viscosities, we used $\mathrm{K}$-junctions where the confluent point is in the junction itself (Fig. 8).

\subsection{Single K-junction}

We first characterize the operation of a single K-junction operated with different combinations of flow rates of the viscous (V) and non-viscous (NV) liquids. For the more viscous liquid, we used a $62 \%(\mathrm{w} / \mathrm{w})$ solution of glycerol in water $\left(\mu=10.11 \times 10^{-3} \mathrm{~Pa}\right.$ s) while a solution of dye in distilled water $\left(\mu=0.89 \times 10^{-3} \mathrm{~Pa} \mathrm{~s}\right)$ served as the less viscous fluid. In order to stabilize the viscosities during the experiments, we stabilized the temperature of the containers and resistive capillaries in a water bath at $25{ }^{\circ} \mathrm{C}$.

As a higher viscosity of the droplet phase promotes the transition to jetting at lower flow rates (Tice et al. 2004;
Berthier et al. 2010), we decided to modify the surface of our microchannels hydrophobically with dodecylamine (Jankowski et al. 2011). As is evident from a comparison of the dotted line in Fig. 9a and the dashed-dotted line in Fig. 4, this modification significantly expanded the range of flow rates that resulted in reproducible dripping.

\subsection{Order of delivery of liquids}

We observed that the range of flow rates that produced droplets depended on the order of delivery of the liquids into the K-junction: (1) the more viscous liquid (V) supplied from above (upstream of) the less viscous (NV) fluid, an arrangement that we refer to as V-NV (Fig. 8c) and (2) the opposite case of the NV liquid supplied from above the V liquid (NV-V) (Fig. 8b). Our screens (Fig. 9) of droplet 

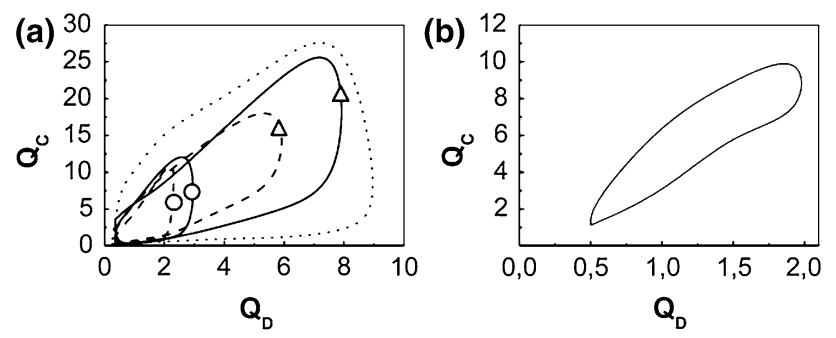

Fig. 9 The regions in the $Q_{D}, Q_{C}$ space that result in the formation of monodisperse droplets in the K-junctions (a). The solid and dashed lines correspond to the $\mathrm{NV}-\mathrm{V}$ and $\mathrm{V}-\mathrm{NV}$ configurations, respectively. The dotted line shows the boundary of the region promoting formation of monodisperse droplets from liquids of the same (low) viscosity. In the same diagram triangles and circles symbolize different ratios of flow of the viscous and less viscous fluids: $Q_{\mathrm{NV}} /$ $Q_{\mathrm{V}}=6 / 1$ and $Q_{\mathrm{NV}} / Q_{\mathrm{V}}=1 / 6$, respectively. b Working range of the chip that simultaneously produces droplets of different ratios $Q_{\mathrm{NV}}$ / $\left(Q_{\mathrm{V}}+Q_{\mathrm{NV}}\right)=\{0,1 / 7,2 / 7,3 / 7,4 / 7,5 / 7,6 / 7, \infty\}$

generation in these two configurations at different combinations of the flow rates of the liquids showed the tendency of narrowing the range of the generation of droplets of increased viscosity, yet the reduction is much more severe in the $\mathrm{V}-\mathrm{NV}$ configuration than in the $\mathrm{NV}-\mathrm{V}$ one. In order to maximize the throughput of the chip, it is better to supply the more viscous liquid from above (stream-wise) the less viscous liquid. This observation has a simple and intuitive explanation: the T-junction systems cross-over into the jetting regime when the liquid adjacent to the wall of the main channel downstream of the junction no longer breaks. An increase in the viscosity of the liquid adjacent to this wall promotes the transition to jetting, and thus it is preferred to keep the more viscous liquid away from the wall.

As is evident from the diagrams shown in Fig. 9a, the maximum rate of droplet formation decreases with increasing fraction of the more viscous liquid. Thus, in the parallel system the maximum rate is limited to the maximum rate of the junction that forms droplets composed entirely of the more viscous liquid (Fig. 9b).
3.6 Volumes of the droplets

In spite of the complication associated with the significantly different viscosities of the input streams, and with the fact that each of the junctions was supplied with a different combination of flow rates of the input streams, the device successfully generated monodisperse droplets (Fig. 10). The dependence of the droplet volume on the flow rates suggests a slight deviation from linearity of $V$ in $Q_{D} / Q_{C}$, yet the ratio of flow rates remains the principal determinant of the volume of the droplets. This result is quite surprising, given the much higher hydrodynamic complexity of formation of droplets from compound streams of liquids significantly differing in viscosity, in comparison to the generation of droplets from homogeneous liquids in a standard T-junction.

\subsection{Volume distribution of the droplets}

The micrograph shown in Fig. 11a suggests that the droplets comprising viscosity gradations are remarkably monodisperse. We quantified the standard deviation of the lengths of the droplets both within the individual channel $(\sigma)$ and across the parallel device $\left(\sigma^{*}\right)$. We find $\sigma<5 \%$ always, while $\sigma^{*}$ is smaller than $5 \%$ at larger flow rates of the continuous liquid.

\subsection{Concentration profiles}

To confirm the designed profile of concentrations, we analyzed the images in a similar way as in the case of the linear and logarithmic gradients operating with liquids of the same viscosity. In Fig. 11b, we illustrate the linear profiles of the concentration in the parallel streams for the lowest and the highest flow rates $\left\{Q_{D}, Q_{C}\right\}(\{4,9\} \mathrm{mL} / \mathrm{h}$ and $\{16,85\}$ $\mathrm{mL} / \mathrm{h}$ ). The droplets maintain, almost perfectly, the desired linear concentration gradation of the dye. The $R^{2}$ values for the gradations that we recorded over a wide range of flow rates all fell above $\sim 0.97$ (Fig. 14 in SI).
Fig. 10 Dependence of the size of the microdroplets on the ratio of the droplet and the continuous phases in the case when fluids varying in viscosity are applied. Micrographs illustrating microdroplets of different size, depending on the applied flow rates (left micrograph $Q_{D}=4 \mathrm{~mL} / \mathrm{h}$, $Q_{C}=16 \mathrm{~mL} / \mathrm{h} ;$ right micrograph $Q_{D}=4 \mathrm{~mL} / \mathrm{h}$, $Q_{C}=9 \mathrm{~mL} / \mathrm{h}$ )
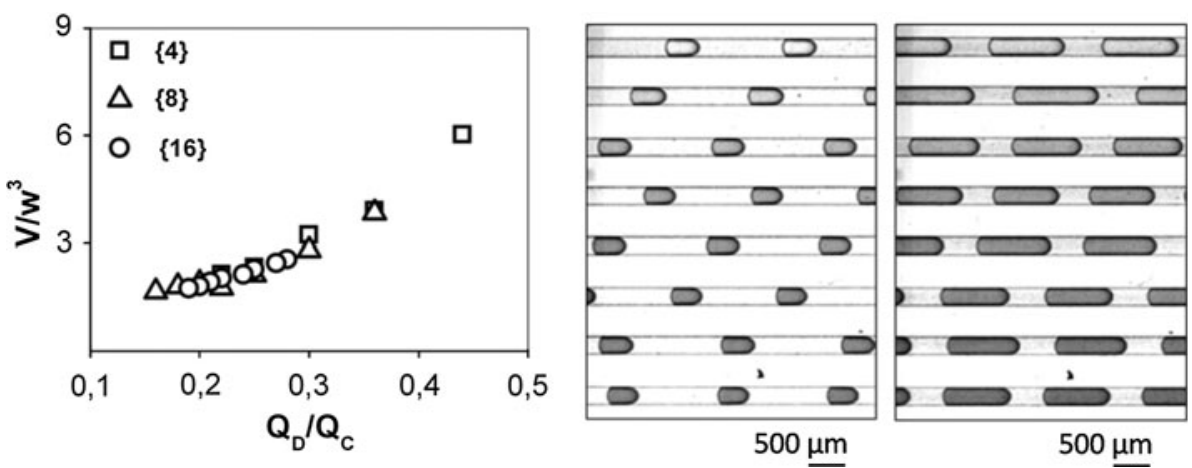

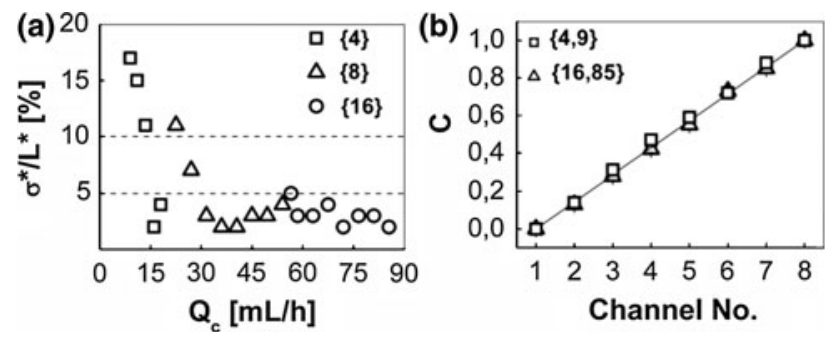

Fig. 11 a The relative standard deviation of the droplet lengths calculated over droplets in all the parallel channels in the device supplied with liquids of different viscosity. The symbols correspond to different flow rates $\left\{Q_{D}\right\}(\mathrm{mL} / \mathrm{h})$. b The recorded (symbols) and desired (solid line) gradations of the dye concentration in the droplets comprising liquids of dissimilar viscosity. The numbers in the brackets give the flow rates $\left\{Q_{D}, Q_{C}\right\}(\mathrm{mL} / \mathrm{h})$

\section{Discussion}

We have described an architecture of the microfluidic chips that allows for (1) the generation of gradations of chemistry in parallel streams of droplets, (2) maximizes the rate of the generation of droplets. Furthermore, our method is time saving with regard to the simultaneity of the droplet creation, and cost limiting as there is no need to use any extra elements such as valves or electronic systems to control the concentration of the applied ingredients. The design that we propose eliminates the necessity of mixing the input streams multiple times, and the dependence of the output on the viscosity of the intermediate mixtures. This, in turn, allowed us to use the same system to produce concentration gradations in droplets comprising liquids of different viscosities.

Our report adds to the studies of the mechanism of the formation of droplets in microfluidic junctions. Our observations suggest that the range of flow rates over which the system produces monodisperse droplets depends on the surface properties. A comparison of the diagrams in Figs. $3 \mathrm{~b}$ and $9 \mathrm{a}$ shows that, even though the unmodified surface of polycarbonate is sufficiently hydrophobic to allow for the generation of droplets of water in hexadecane, an increase of the hydrophobic character, via a modification of PC with dedecylamin, significantly expands the range that promotes dripping. In other words, the transition from dripping to jetting seems to depend on the value of the contact angle of the dispersed phase on the surface (in the air $84^{\circ}$ for water on native PC and $130^{\circ}$ on PC modified with DDA; in the $2 \%$ solution of SPAN80 in hexadecane $105.3^{\circ}$ on native and $139.7^{\circ}$ on modified surface).

The second fundamental observation is that in the systems that break compound streams of liquids of different viscosities into droplets, the range of flow rates that promotes dripping depends on the order of delivery of the less and more viscous liquids. Having the more viscous liquid separated from the wall onto which the neck of the droplet collapses during the break-up, shifts the transition to jetting to higher flow rates.

The systems that we have demonstrated here produced linear and logarithmic concentration gradations in the droplets. All these systems formed tightly monodisperse droplets, and faithfully represented the designed concentration gradations over a wide range of flow rates. This allowed us to (1) tune the volume of the droplets over a wide range (from 5 to $80 \mathrm{~nL}$ ) and to (2) maximize the throughput of the system up to ca. $6 \mathrm{~mL} / \mathrm{h}$ per single junction, while applying non-viscous solutions (water) or $2 \mathrm{~mL} / \mathrm{h}$ in the case of an arrangement concerning liquids of different viscosities.

One potential drawback of the architecture that we propose here, in comparison to the systems that mix the input streams at different combinations in a network of interconnected channels, is that the maximum ratio of output concentrations in the droplets translates into a ratio of resistances of the channels that deliver input streams into the confluent point. In designs that keep the cross sections of all the channels similar, this condition makes it impractical to produce gradations comprising ratios spanning over many orders of magnitude. With precise fabrication schemes it is, however, possible to obtain a large span of resistances of the delivery channels by varying their cross sections. Importantly, the architectures that we propose here can be easily and freely modified to generate parallel streams of virtually any functional form of concentration gradation, and-at the same time- to produce droplets of different, designed, volume at each of the junctions.

The fact that it is possible to produce parallel streams of drops comprising liquids of different viscosities may make this strategy attractive for preparative applications that use solutions of polymers to form beads or capsules. Potential use of suspensions of spheres of a well-defined distribution of diameters and concentrations of active substances may be useful in cosmetic or pharmaceutical industries to precisely design and control the profiles of release (Xu et al. 2009).

Acknowledgments The Project was operated within the Foundation for Polish Science Team Programme and co-financed by the EU within the European Regional Development Fund, through the grant Innovative Economy (POIG.01.01.02-00-008/08)

Open Access This article is distributed under the terms of the Creative Commons Attribution License which permits any use, distribution, and reproduction in any medium, provided the original author(s) and the source are credited.

\section{References}

Anna SL, Bontoux N, Stone HA (2003) Formation of dispersions using "flow focusing" in microchannels. Appl Phys Lett 82:364-366. doi:10.1063/1.1537519 
Berthier J, Le Vot S, Tiquet P, David N, Lauro D, Benhamou PY, Rivera F (2010) Highly viscous fluids in pressure actuated flow focusing devices. Sens Actuators A Phys 158:140-148. doi: 10.1016/j.sna.2009.12.016

Campbell K, Groisman A (2007) Generation of complex concentration profiles in microchannels in a logarithmically small number of steps. Lab Chip 7:264-272. doi:10.1039/b610011b

Cao JL, Kursten D, Schneider S, Knauer A, Gunther PM, Kohler JM (2012) Uncovering toxicological complexity by multi-dimensional screenings in microsegmented flow: modulation of antibiotic interference by nanoparticles. Lab Chip 12:474-484. doi:10.1039/c1lc20584f

Christopher GF, Anna SL (2007) Microfluidic methods for generating continuous droplet streams. J Phys D Appl Phys 40:R319-R336. doi:10.1088/0022-3727/40/19/r01

Christopher GF, Noharuddin NN, Taylor JA, Anna SL (2008) Experimental observations of the squeezing-to-dripping transition in T-shaped microfluidic junctions. Phys Rev E 78. doi: 10.1103/PhysRevE.78.036317

Chu LY, Utada AS, Shah RK, Kim JW, Weitz DA (2007) Controllable monodisperse multiple emulsions. Angew Chem Int Ed 46:8970-8974. doi:10.1002/anie.200701358

Churski K, Korczyk P, Garstecki P (2010) High-throughput automated droplet microfluidic system for screening of reaction conditions. Lab Chip 10:816-818. doi:10.1039/b925500a

Damean N, Olguin LF, Hollfelder F, Abell C, Huck WTS (2009) Simultaneous measurement of reactions in microdroplets filled by concentration gradients. Lab Chip 9:1707-1713. doi:10.1039/ b821021g

Dertinger SKW, Chiu DT, Jeon NL, Whitesides GM (2001) Generation of gradients having complex shapes using microfluidic networks. Anal Chem 73:1240-1246. doi:10.1021/ac001 $132 \mathrm{~d}$

Dertinger SKW, Jiang XY, Li ZY, Murthy VN, Whitesides GM (2002) Gradients of substrate-bound laminin orient axonal specification of neurons. Proc Nat Acad Sci USA 99:12542-12547. doi:10.1073/pnas.192457199

Derzsi L, Jankowski P, Lisowski W, Garstecki P (2011) Hydrophilic polycarbonate for generation of oil in water emulsions in microfluidic devices. Lab Chip 11:1151-1156. doi:10.1039/c0lc $00438 \mathrm{c}$

Garstecki P, Stone HA, Whitesides GM (2005) Mechanism for flowrate controlled breakup in confined geometries: a route to monodisperse emulsions. Phys Rev Lett 94. doi: 10.1103/Phys RevLett.94.164501

Garstecki P, Fuerstman MJ, Stone HA, Whitesides GM (2006) Formation of droplets and bubbles in a microfluidic T-junctionscaling and mechanism of break-up. Lab Chip 6:437-446. doi: $10.1039 / \mathrm{b} 510841 \mathrm{a}$

Guillot P, Colin A (2005) Stability of parallel flows in a microchannel after a T junction. Phys Rev E 72. doi:10.1103/PhysRevE.72.06 6301

Guillot P, Ajdari A, Goyon J, Joanicot M, Colin A (2009) Droplets and jets in microfluidic devices. C R Chim 12:247-257. doi: 10.1016/j.crci.2008.07.005

Guzowski J, Korczyk PM, Jakiela S, Garstecki P (2011) Automated high-throughput generation of droplets. Lab Chip 11:3593-3595. doi:10.1039/c1lc20595a

Hattori K, Sugiura S, Kanamori T (2009) Generation of arbitrary monotonic concentration profiles by a serial dilution microfluidic network composed of microchannels with a high fluidic-resistance ratio. Lab Chip 9:1763-1772. doi:10.1039/b816995k

Husny J, Cooper-White JJ (2006) The effect of elasticity on drop creation in T-shaped microchannels. J Non Newton Fluid Mech 137:121-136. doi:10.1016/j.jnnfm.2006.03.007
Irimia D, Liu SY, Tharp WG, Samadani A, Toner M, Poznansky MC (2006) Microfluidic system for measuring neutrophil migratory responses to fast switches of chemical gradients. Lab Chip 6:191-198. doi:10.1039/b511877h

Jankowski P, Ogonczyk D, Kosinski A, Lisowski W, Garstecki P (2011) Hydrophobic modification of polycarbonate for reproducible and stable formation of biocompatible microparticles. Lab Chip 11:748-752. doi:10.1039/c0lc00360c

Kenis PJA, Ismagilov RF, Whitesides GM (1999) Microfabrication inside capillaries using multiphase laminar flow patterning. Science 285:83-85. doi:10.1126/science.285.5424.83

Kim C, Lee K, Kim JH, Shin KS, Lee K-J, Kim TS, Kang JY (2008) A serial dilution microfluidic device using a ladder network generating logarithmic or linear concentrations. Lab Chip 8:473-479. doi:10.1039/b714536e

Korczyk PM, Cybulski O, Makulska S, Garstecki P (2011) Effects of unsteadiness of the rates of flow on the dynamics of formation of droplets in microfluidic systems. Lab Chip 11:173-175. doi: $10.1039 / \mathrm{c} 01 \mathrm{c} 00088 \mathrm{~d}$

Lee JN, Park C, Whitesides GM (2003) Solvent compatibility of poly(dimethylsiloxane)-based microfluidic devices. Anal Chem 75:6544-6554. doi:10.1021/ac0346712

Lee K, Kim C, Kim Y, Jung K, Ahn B, Kang JY, Oh KW (2010) 2-layer based microfluidic concentration generator by hybrid serial and volumetric dilutions. Biomed Microdevices 12:297-309. doi:10.1007/s10544-009-9385-6

Lee W, Walker LM, Anna SL (2011) Competition between viscoelasticity and surfactant dynamics in flow focusing microfluidics. Macromol Mater Eng 296:203-213. doi:10.1002/mame.201000302

Li W, Nie Z, Zhang H, Paquet C, Seo M, Garstecki P, Kumacheva E (2007) Screening of the effect of surface energy of microchannels on microfluidic emulsification. Langmuir 23:8010-8014. doi:10.1021/la7005875

Lin F, Saadi W, Rhee SW, Wang SJ, Mittal S, Jeon NL (2004) Generation of dynamic temporal and spatial concentration gradients using microfluidic devices. Lab Chip 4:164-167. doi: $10.1039 / \mathrm{b} 313600 \mathrm{k}$

Lorenz RM, Fiorini GS, Jeffries GDM, Lim DSW, He M, Chiu DT (2008) Simultaneous generation of multiple aqueous droplets in a microfluidic device. Anal Chim Acta 630:124-130. doi: 10.1016/j.aca.2008.10.009

Miller OJ, El Harrakb A, Mangeatb T, Bareta J-Ch, Frenza L, El Debsa B, Mayota E, Samuelsc ML, Eamonn K, Rooneyd E, Dieue P, Galvand M, Linkc DR, Griffithsa AD (2012) Highresolution dose-response screening using droplet-based microfluidics. PNAS 109:378-383. doi:10.1073/pnas.1113324109

Neils C, Tyree Z, Finlayson B, Folch A (2004) Combinatorial mixing of microfluidic streams. Lab Chip 4:342-350. doi:10.1039/b314962e

Nie Z, Seo M, Xu S, Lewis PC, Mok M, Kumacheva E, Whitesides GM, Garstecki P, Stone HA (2008) Emulsification in a microfluidic flow-focusing device: effect of the viscosities of the liquids. Microfluid Nanofluid 5:585-594. doi:10.1007/s10404008-0271-y

Nisisako T, Torii T (2008) Microfluidic large-scale integration on a chip for mass production of monodisperse droplets and particles. Lab Chip 8:287-293. doi:10.1039/b713141k

Niu X, Gielen F, Edel JB, deMello AJ (2011) A microdroplet dilutor for high-throughput screening. Nature Chem 3:437-442. doi: 10.1038/NCHEM.1046

Ogonczyk D, Wegrzyn J, Jankowski P, Dabrowski B, Garstecki P (2010) Bonding of microfluidic devices fabricated in polycarbonate. Lab Chip 10:1324-1327. doi:10.1039/b924439e

Seo M, Nie ZH, Xu SQ, Mok M, Lewis PC, Graham R, Kumacheva E (2005) Continuous microfluidic reactors for polymer particles. Langmuir 21:11614-11622. doi:10.1021/la050519e 
Song H, Chen DL, Ismagilov RF (2006) Reactions in droplets in microfluidic channels. Angew Chem Int Ed 45:7336-7356. doi: 10.1002/anie.200601554

Thorsen T, Roberts RW, Arnold FH, Quake SR (2001) Dynamic pattern formation in a vesicle-generating microfluidic device. Phys Rev Lett 86:4163-4166. doi:10.1103/PhysRevLett.86.4163

Tice JD, Lyon AD, Ismagilov RF (2004) Effects of viscosity on droplet formation and mixing in microfluidic channels. Anal Chim Acta 507:73-77. doi:10.1016/j.aca.2003.11.024

Utada AS, Lorenceau E, Link DR, Kaplan PD, Stone HA, Weitz DA (2005) Monodisperse double emulsions generated from a microcapillary device. Science 308:537-541. doi:10.1126/science. 1109164

van Steijn V, Kleijn CR, Kreutzer MT (2010) Predictive model for the size of bubbles and droplets created in microfluidic T-junctions. Lab Chip 10:2513-2518. doi:10.1039/c002625e

Xu SQ, Nie ZH, Seo M, Lewis P, Kumacheva E, Stone HA, Garstecki P, Weibel DB, Gitlin I, Whitesides GM (2005) Generation of monodisperse particles by using microfluidics: control over size, shape, and composition. Angew Chem Int Ed 44:724-728. doi: 10.1002/anie. 200462226
Xu Q, Hashimoto M, Dang TT, Hoare T, Kohane DS, Whitesides GM, Langer R, Anderson DG (2009) Preparation of monodisperse biodegradable polymer microparticles using a microfluidic flow-focusing device for controlled drug delivery. Small 5:1575-1581. doi:10.1002/smll.200801855

Yamada M, Hirano T, Yasuda M, Seki M (2006) A microfluidic flow distributor generating stepwise concentrations for high-throughput biochemical processing. Lab Chip 6:179-184. doi:10.1039/ b514045d

Yeom S, Lee SY (2011) Size prediction of drops formed by dripping at a micro T-junction in liquid-liquid mixing. Exp Thermal Fluid Sci 35:387-394. doi:10.1016/j.expthermflusci.2010.10.009

Yusuf HA, Baldock SJ, Fielden PR, Goddard NJ, Mohr S, Brown BJT (2010) Systematic linearisation of a microfluidic gradient network with unequal solution inlet viscosities demonstrated using glycerol. Microfluid Nanofluid 8:587-598. doi:10.1007/ s10404-009-0489-3

Zheng B, Gerdts CJ, Ismagilov RF (2005) Using nanoliter plugs in microfluidics to facilitate and understand protein crystallization. Curr Opin Struct Biol 15:548-555. doi:10.1016/j.sbi.2005. 08.009 